\title{
Large air pressure changes triggered by P-SV ground motion in a cave in northern Taiwan
}

Chieh-Hung Chen ( $\nabla$ nononochchen@gmail.com )

China University of Geosciences

\section{Yang-Yi Sun}

China University of Geosciences

\section{Li-Ching Lin}

Science and Technology policy research and information center

\section{Peng Han}

Southern University of Science and Technology

\section{Huai-Zhong Yu}

China Earthquake Networks Center

\section{XueMin Zhang}

China Earthquake Administration

\section{Chi-Chia Tang}

China University of Geosciences

\section{Chun-Rong Chen}

National Central University

Horng-Yuan Yen

National Central University

Cheng-Horng Lin

Academia Sinica

Jann-Yenq Liu

National Central University

Ching-Ren Lin

Academia Sinica

\section{Research Letter}

Keywords: Air pressure, Pressure-Shear vertical waves, Acoustic waves

Posted Date: January 5th, 2021

DOI: https://doi.org/10.21203/rs.3.rs-53321/v2 
License: (c) (i) This work is licensed under a Creative Commons Attribution 4.0 International License. Read Full License

Version of Record: A version of this preprint was published at Scientific Reports on June 18th, 2021. See the published version at https://doi.org/10.1038/s41598-021-92216-w. 


\section{Abstract}

A barometer in the cave of the SBCB station records an unusual phenomenon of larger amplitudes in air pressure changes inside than those at the Xinwu station (outside). Accordingly, the comparison between the recorded data at the SBCB and Xinwu station can drive investigations of potential sources of the unusual phenomenon. Analytical results of phase angle differences reveal that the air pressure outside the cave at the Xinwu station often leads air pressure changes inside at the SBCB station at relatively low frequency bands. In contrast, the larger pressure changes at frequencies $>2 \times 10^{-4} \mathrm{~Hz}$ inside the cave at the SBCB station lead smaller changes outside at the Xinwu station. To expose causal mechanisms of the unusual phenomenon, continuous seismic waveforms recorded inside the cave at the SBCB station are further conducted for examination. When the horizontal and vertical ground velocities of ground motion yield a difference in the phase angle close to $90^{\circ}$, amplitudes of the air pressure changes at the SBCB station are amplified accordingly. This suggests that the pressure-shear vertical ground motion can drive air pressure changes. Meanwhile, the results shed light on investigating the existence of acoustic waves near the Earth's surface using a partially confined space underground due to that the assumptions of the waves can propagate upward in the atmosphere driving changes in the ionosphere.

\section{Introduction}

A barometer is one of scientific instruments that is generally installed above the Earth's surface and is utilized to monitor variations of atmospheric pressure in a particular environment (Kenneth, 2013). The monitoring collects useful information (i.e., atmospheric pressure) to help weather analysis and to forecast short-term changes in the weather for further evaluating impacts on human life (Rasouli et al., 2012; Gutierrez-Lopez et al., 2019). Alternatively, the observation exhibits low-noise characteristics in caves and/or tunnels with rare artificial activities due to that the environment inside can mitigate influence from weather and artificial activities outside. The recorded data can be utilized as references for correcting responses of air pressure on distinct geophysical measurements (Huang et al., 2009) and detecting an unlike change in deep tunnels that is resulted from the weather through a comparison with outside (Wasilewski, 2014; Tan et al., 2008).

Previous studies (Liu et al., 2006, 2011a, 2011b, 2016, 2019; Otsuka et al., 2009; Astafyeva et al., 2011; Sun et al., 2011, 2016; Astafyeva, 2019; Chou et al., 2020) reported that acoustic wavs can propagate from the ground upward the atmosphere and drives changes in electron density in the ionosphere. However, acoustic wavs originate from ground vibrations that is obscured and difficult to be identified. The difficulty is mainly due to that seismic waves generally comprises distinct types of ground vibrations. Meanwhile, the acoustic wavs disperse in an open area (i.e., near the Earth's surface) and become weak, accordingly.

A cave of the SBCB station is located at $\left(24.79^{\circ} \mathrm{N}, 120.98^{\circ} \mathrm{E}\right)$ with the altitude of $\sim 141.5$ meters beneath the Eighteen-Peaks Mountain in the northeastern Taiwan. The cave was built for bomb shelter in 1941 (https://gps.moi.gov.tw/SSCenter/Introduce/IntroducePage.aspx?Page=Gravity4). Aisles inside the cave 
exhibit as a "U" sharp. The cave wall is rocks covered by calcium silicate boards and tiles. The width and the height of the cave is about 1.2-1.5 meters and 1.8 meters, respectively. The cave is under overburden soils with a thickness of about 40 meters that causes the stable temperature and structural integrity (Huang, et al., 2009). Meanwhile, the influence from the groundwater is relatively small due to that the groundwater is static and its level is about 23 meters lower than the cave. Doors were set up in the both entrances of the cave to avoid the interference from artificial activities. A broadband seismometer was installed at the innermost of the cave (Fig. S1-S4), where is about 38 meters away from the entrances, due to that the interior of the cave can eliminate the effects of the artificial activity and severe weather outside the cave. A barometer of the Setra's Model 278 with an accuracy of $\sim 0.3 \mathrm{mb}$ was installed beside a broadband seismometer (Fig. S5) for correcting unwanted influence from air pressure changes on seismic data (Beauduin et al., 1996; Zurn et al., 2007). All the efforts are benefit to high-quality data (Huang, et al., 2009). Note that the other barometer of the Setra's Model 270 with an accuracy of about $0.05 \%$ of the full scale was installed at the Xinwu (operated by the Central Weather Bureau in Taiwan code 467050$)$ weather station $\left(25.00^{\circ} \mathrm{N}, 121.05^{\circ} \mathrm{E}\right.$; Fig. 1a) with the altitude of $\sim 20$ meters, where is approximately $25 \mathrm{~km}$ away from the cave. The barometer was set a few meters above the Earth's surface for routinely monitoring changes in air pressure dominated by the weather.

Figure $1 \mathrm{~b}$ shows air pressure changes inside and outside the cave from February 1 to 5 2016. The air pressure outside the cave at the Xinwu station is mainly ranged from $1016 \mathrm{mb}$ to $1024 \mathrm{mb}$. Alternatively, the air pressure inside the cave at the SBCB station is ranged from $1004 \mathrm{mb}$ to $1012 \mathrm{mb}$. Air pressure at the Xinwu and SBCB station roughly shows a discrepancy of about $12 \mathrm{mb}$. The discrepancy is considered to be contributed by difference of altitudes ( $121=141-20$ meters) between these two barometers based on the decrease rate ( $1 \mathrm{mb} / 9$ meters) close to the Earth's surface. Air pressure data show the semidiurnal variations and exhibit in-phase changes at the Xinwu and SBCB stations (Fig. 1b). Amplitudes of noise at both stations are about $0.1 \mathrm{mb}$. According to the comparison, amplitudes of the pressure perturbations at the SBCB station are roughly comparable from February 1 to 3 in 2016. In contrast, the amplitudes are approximately $0.5 \mathrm{mb}$ greater than those at the Xinwu station particularly from February 4 to 5 in 2016 (red arrows in Fig. 1b as examples). The great amplitude inside the cave is an unusual phenomenon due to that artificial activities are rare, which leads us to investigate a causal mechanism of the large pressure changes at the SBCB station.

\section{Methodology And Analytical Results}

To examine the causal mechanism of the characteristics including the amplitude and the frequency, we transformed both air pressure datasets into the frequency domain using the Fourier transform. We also assessed the coherence of the amplitude within a particular frequency band using the Magnitude-Square Coherence (MSC) index (Stoica \& Randolph, 2005). The MSC was computed from the bivariate time series using a subroutine called "mscohere" in MATLAB. The MSC can identify significant frequencydomain correlations between two time series datasets. Meanwhile, phase angle estimates in the cross spectrum are useful for understanding where significant frequency-domain correlations exist. The 
differences in the phase angles of a particular frequency band were computed to understand the leading (or lagging) of air pressure changes at the Xinwu and SBCB station.

The air pressure data at the Xinwu and SBCB stations have coherence values close to 1 near a frequency of $3 \times 10^{-5} \mathrm{~Hz}$ ( semi-diurnal; Fig. 1c). The green circles at the particular frequency band in Fig. $1 \mathrm{c}$ reveal that variations in air pressure outside the cave at the Xinwu station occurred before (leaded) variations in air pressure inside the cave at the SBCB station. In normal, under typical conditions, the barometer takes about 1 minute to respond to weather changes outside the cave (Fig. S6) at frequencies $<1 \times 10^{-4} \mathrm{~Hz}$ (Fig. 1c). On the other hand, the coherence rapidly decreases a relatively low stage at frequencies $>2 \times 10^{-4} \mathrm{~Hz}$ (Fig. 1c). Red circles with the coherences $>\sim 0.4$ can be frequently observed at frequencies $>2 \times 10^{-4} \mathrm{~Hz}$ (Fig. 1C). This suggests that variations in air pressure at the Xinwu and SBCB station are similar. The variations at the Xinwu station lag behind the changes at the SBCB station. The perturbations inside the cave at the SBCB station sometimes lead perturbations outside it at the Xinwu station (i.e., red circles), which is entirely different from our common senses that the pressure variations should be relatively-small and quiet inside, if the variations inside are due to weather and human activity outside.

The odds ratio (Holcomb, 2001; Viera, 2008, Szumilas, 2010) is defined as $p /(1-p)$ where $p$ is the probability of success and is used in this study to show if the leading events were statistically significant. Notably, with an odds ratio near one, a success (i.e., an enhancement in this study) was more likely than a failure. We calculated the odds ratios by dividing the number of the leading events by the number of the lagging ones within a moving window of 5 events. The odds ratios are obviously larger than one in particular frequency bands (e.g., close to $1 \times 10^{-4} \mathrm{~Hz}, 4 \times 10^{-4}-7 \times 10^{-4} \mathrm{~Hz}, 1 \times 10^{-3} \mathrm{~Hz}, 1.7 \times 10^{-3}, 4 \times 10^{-3} \mathrm{~Hz}$, $6 \times 10^{-3}$ and $8 \times 10^{-3} \mathrm{~Hz}$ in Fig. $1 \mathrm{~d}$ ). This suggests that those promising leading events can pass the statistical test (i.e., the odds ratio $>1$ ) and exist in the observation data in particular frequency bands. In short, we found that air pressure with an amplitude of approximately $0.5 \mathrm{mb}$ at the SBCB station is larger than it at the Xinwu station. Variations of the air pressure at the SBCB station is mainly dominated by those outside the cave for the relatively-low frequency band $\left(<2 \times 10^{-4} \mathrm{~Hz}\right)$. In contrast, for the relativelyhigh frequency band $\left(>2 \times 10^{-4} \mathrm{~Hz}\right)$, variations of the air pressure at the SBCB station can lead them at the Xinwu station. The large amplitude at the SBCB station is mainly limited within the relatively-high frequency band.

\section{Discussions}

Wasilewski (2014) and Tan et al. (2008) reported that the amplification effect of air pressure changes in deep mines can be caused by variations of the environments on the Earth's surface due to the partially confined interior of tunnels. However, the amplified variations of the air pressure reported in this study are observed in the shallow cave with short aisles. Variations of the environments outside the caves are not the major factor of the large changes of the air pressure observed inside in this study. The variations of the air pressure at the SBCB station lead those at the Xinwu station in the relatively-high frequency band of $>2 \times 10^{-4} \mathrm{~Hz}$ (Fig. 1C). This suggests the air can be squeezed out from the cave in the relatively-high 
frequency band. Even the air can be squeezed out from the cave, the pressure perturbations at the Xinwu station caused by air blowing remains questionable due to that the difference of $0.5 \mathrm{mb}$ becomes smaller with the propagation via dispersion.

Previous studies (Artru et al., 2004; Hao et al., 2013; Liu et al., 2016) reported that changes in air pressure can be triggered by the arrival of propagating Rayleigh-like (Pressure-Shear vertical; P-SV) waves. Thus, beside the air blowing in the atmosphere, large-scale ground motion forces the ground and perturbs the air that can be one of the candidates for resulting the lags. The large-scale ground motion amplifies variations in air pressure changes inside the cave due to the confinement of the surrounding rocks and influences surface air pressure. In other words, relatively-large variations should result from activities inside the cave or beneath the ground that shows the possible connection between changes in ground motion and air pressure changes.

To examine the connection, continuous seismic waveforms (i.e., seismic data) were also analyzed in this study to understand how ground motion triggers air pressure variations. We computed the coherence and the phase angle difference varying with frequencies between the vertical ground velocity (Fig. 2a) and the air pressure at both the SBCB and Xinwu stations. A low coherence close to 0.2 in most of the frequency bands (Fig. 2b) suggests that, in a typical condition, changes in air pressure are generally uncorrelated to ground motion. However, the ground motion leads to changes in air pressure at the SBCB station (red circles in Fig. 2b), which can be observed in the frequency bands (e.g., close to $4 \times 10^{-4}-7 \times 10^{-4} \mathrm{~Hz}, 1.5 \times 10^{-}$ ${ }^{3}-4 \times 10^{-3} \mathrm{~Hz}, 6 \times 10^{-3}-7 \times 10^{-3} \mathrm{~Hz}$ in Fig. $2 \mathrm{~d}$ ) that exhibits the higher coherences $(>0.35)$. The higher coherence suggests that the ground motion and the air pressure in the cave exhibit relatively similar variations at the particular frequency bands. Changes of the air pressure in the cave are probably dominated by the ground motion at the SCBC station at these particular frequency bands. We thus investigate whether air pressure at the Xinwu station changes accordingly or dissipates due to dispersion.

We distinguished changes in air pressure at the Xinwu station that are driven by ground motion using the same method (i.e., coherence and phase angle differences). Similarly, changes in air pressure at the Xinwu station are almost uncorrelated with ground motion, except for several specific frequencies close to $3 \times 10^{-4}-9 \times 10^{-4} \mathrm{~Hz}, 1.5 \times 10^{-3}-4 \times 10^{-3} \mathrm{~Hz}$, and $6 \times 10^{-3}-7 \times 10^{-3} \mathrm{~Hz}$ (Figs. $2 \mathrm{c}$ and $2 \mathrm{e}$ ). In these frequency bands, we can find that ground motion leads changes in air pressure at the Xinwu station exhibiting the higher coherence $(>0.35)$.

One of the characteristics associated with P-SV waves is the $90^{\circ}$ phase angle difference between the horizontal and vertical components. We computed the maximum horizontal amplitude as the horizontal component (Fig. 2a) by using the East-West and North-South ground velocities utilizing the method proposed by Tanimoto et al. (2006). We determine the ground motion with the differences of the phase angle between the horizontal and the vertical component are from $75^{\circ}$ to $105^{\circ}$ and from $-105^{\circ}$ to $-75^{\circ}$ as P-SV waves. We integrated coherences that were larger than distinct thresholds ranging from 0 to 0.35 and counted the numbers associated with the P-SV waves for the different criteria. The upper bound of the threshold is determined by 0.35 due to insufficient numbers of the coherences $>0.35$. The P-SV 
motion ratios (a number of the P-SV waves at each frequency grid / a total number of the frequency grids) are proportional to the coherence, and increase to double the average (i.e., $0.17=60 / 360$ ) for a threshold of 0.35 (Fig. 2f). Analytical results suggest that when the P-SV waves dominate ground motion, air pressure and the ground motion share frequencies exhibiting similar variations.

We try to evaluate air pressure changes dominated by variations of the volume of the cave through the ideal gas law (Clapeyron, 1834). We assumed that the total number of moles and temperature of the air inside the cave are constant, while ground vibrations trigger changes in air pressure without break and/or damage. The volume of the cave in this study is approximately $270.00(=1.5$ in width $x 1.8$ in height $x 100$ in length) $\mathrm{m}^{3}$. If the P-SV ground vibrations contribute variations of $\pm 0.25 \mathrm{mb}$ in air pressure, the changes of the volume are about $0.14 \mathrm{~m}^{3}$, accordingly, for maintaining the product of the air pressure and the volume. If the changes of the volume are mainly contributed by the vertical component of the vibrations, the amplitude of the ground vibrations in the cave is about $10^{-3}$ meters. The comparable results between the observation and the model suggest the large air pressure changes in a cave can be attributed to the PSV-type ground vibrations.

If the P-SV ground motion can drive changes in air pressure, the question is how often the interaction can be detected. The interaction of events by using both the P-SV ground motion and a coherence value > 0.35 at each particular frequency can be determined. The total number of interaction events was generally maintained at 20 during the study period of 930 days (from January 1, 2015 to July 19, 2018; in Fig. 3). This finding suggests that interactions permanently occur every day. These interactions can be dominated by the P-SV microseisms triggered by the interaction between oceanic waves and land (e.g., Cessaro, 1994; Kimman et al., 2012).

\section{Conclusion}

This study proposes an efficient method to document the physical evidence of the P-SV type ground motion triggering changes in air pressure near the Earth's surface. When the ground motion with the P-SV type is related to microseisms, the air pressure can change accordingly. The air pressure in caves can be amplified by the existence of the P-SV type motion due to the interior space being partially confined (similar to press a rubber air ball). Thus, the air pressure retrieved from a barometer inside a cave is sensitive to the P-SV type motion.

The novel observation sheds light on extension of the use of a cave and/or a tunnel. The amplified air pressure triggered by the P-SV type motion creates an excellent opportunity to study the origin of acoustic waves from ground motion. Air pressure data observed in a cave and/or a tunnel can become a treasure, while scientists want to prove the existence of acoustic waves that propagate upward and drive changes in the atmosphere. On the other hand, the P-SV type ground motion can be often observed in microseisms and surface waves after earthquake occurrence. When stable environments of air pressure are seriously concerned in a cave, the effects of the P-SV type ground motion have to be taken into consideration. 


\section{Declarations}

\section{Availability of data and materials}

Seismic waveform data and air pressure data at SBCB station were provided by the Institute of Earth Sciences, Academia Sinica, Taiwan. The air pressure data at Xinwu station were provided by the Central Weather Bureau, Taiwan. Those data can be downloaded at the website of https://doi.org/10.5061/dryad.05qfttdzh.

\section{Competing interests}

The authors declare that they have no known competing interests that could have appeared to influence the work reported in this paper

\section{Funding}

This research was funded by the Joint Funds of the National Natural Science Foundation of China (Grant no. U2039205), the National Key Research and Development Project (Grant no. 2018YFE0109700), Ministry of Science and Technology of Taiwan (Grants no. MOST 106-2116-M-194-016- and MOST 1062628-M-008-002), and the Sichuan earthquake Agency-Research Team of GNSS based on geodetic tectonophysics and mantle-crust dynamics in the Chuan-Dian region (Grant no. 201803). Meanwhile, this work was also supported by the Center for Astronautical Physics and Engineering (CAPE) from the Featured Area Research Center program within the framework of Higher Education Sprout Project by the Ministry of Education (MOE) in Taiwan.

\section{Author contribution}

Y.Y.S. contributed discussion and revision; L.C.L. contributed discussion and revision; P.H. contributed discussion; H.Z.Y. contributed discussion; X.Z. contributed discussion; C.C.T. contributed data collection; C.R.C. contributed data collection and discussion; H.Y.Y contributed data collection and discussion; C.H.L. contributed data collection, discussion and revision; J.Y.L. contributed discussion and revision; L.C.R. contributed data collection.

\section{Acknowledgements}

The authors appreciate the scientists who devoted their time to maintain instruments in the field and in office data centers that led to the discovery of interesting geophysical phenomena.

\section{References}

Artru, J., Farges, T., Lognonné, P., 2004. Acoustic waves generated from seismic surface waves: Propagation properties determined from Doppler sounding observations and normal-mode modelling. Geophys. J. Int. 158, 1067-1077. 
Astafyeva, E., 2019. Ionospheric Detection of Natural Hazards. Rev. Geophys. 57(4), 1265-1288, doi:10.1029/2019rg000668.

Astafyeva, E., Lognonne, P., Rolland, L., 2011. First ionospheric images of the seismic fault slip on the example of the Tohoku-oki earthquake. Geophys. Res. Lett. 38, L22104, doi:10.1029/2011 gl049623.

Beauduin, R., Lognonne, P., Montagner, J.P., Cacho, S., Karczewski, J.F. and Morand, J., 1996. The effects of the atmospheric pressure changes on seismic signals or how to improve the quality of a station. Bull. Seismol. Soc. Am. 86, 1760-1769.

Chou, M.Y., Cherniak, I., Lin, C.C.H., Pedatella, N.M., 2020. The Persistent lonospheric Responses Over Japan After the Impact of the 2011 Tohoku Earthquake. Space Weather, 18(4), doi: $10.1029 / 2019$ SW002302.

Clapeyron, E., 1834. Mémoire sur la puissance motrice de la chaleur. Journal de l'École Polytechnique (in French). XIV: 153-90.

Gutierrez-Lopez, A., Cruz-Paz, I. \& Muñoz Mandujano, M., 2019. Algorithm to Predict the Rainfall Starting Point as a Function of Atmospheric Pressure, Humidity, and Dewpoint. Climate, 7, 131.

Hao, Y.Q., Xiao, Z., Zhang, D.H., 2013. Teleseismic magnetic effects (TMDs) of 2011 Tohoku earthquake. J. Geophys. Res. 118, 3914-3923.

Holcomb, W., 2001. An odd measure of risk: Use and misuse of the odds ratio. Obstet. Gynecol. 98, (4), 685-688.

Hwang, C., Kao, R., Cheng, C.-C., Huang, J.-F., Lee, C.-W., Sato, T., 2009. Results from parallel observations of superconducting and absolute gravimeters and GPS at the Hsinchu station of Global Geodynamics Project, Taiwan. J. Geophys. Res. 114, B07406.

Rideout, M.S. Kenneth, 2015. Barron's AP Physics[M]凶Barron's Educational Series囚p.p. 313.

Liu, J.Y., Chen, C.H., Lin, C.H., Tsai, H.F., Chen, C.H., Kamogawa, M., 2011b. Ionospheric disturbances triggered by the 11 March 2011 M9.0 Tohoku earthquake. J. Geophys. Res. 116, A06319.

Liu, J.Y., Chen, C.H., Sun, Y.Y., Chen, C.H., Tsai, H.F., Yen, H.Y., Chum, J., Lastovicka, J., Yang, Q.S., Chen, W.S., Wen, S., 2016. The vertical propagation of disturbances triggered by seismic waves of the 11 March2011 M9.0 Tohoku earthquake over Taiwan. Geophys. Res. Lett. 43, 1759-1765, doi:10.1002/2015GL067487.

Liu, J.Y., Chen, C.H., Sun, Y.Y., Chen, C.H., Tsai, H.F., Yen, H.Y., Chum, J. Chum, Lastovicka, J., Yang, Q.S., Chen, W.S., Wen, S., 2016. The vertical propagation of disturbances triggered by seismic waves of the 11 March 2011 M9.0 Tohoku earthquake over Taiwan. Geophys. Res. Lett. 43(4), 1759-1765. doi:10.1002/2015gl067487. 
Liu, J.Y., Chen, C.Y., Sun, Y.Y., Lee, I.T., Chum, J., 2019. Fluctuations on vertical profiles of the ionospheric electron density perturbed by the March 11, 2011 M9.0 Tohoku earthquake and tsunami. GPS Solut. 23(3). doi:10.1007/s10291-019-0866-7.

Liu, J.Y., Sun, Y.Y., 2011a. Seismo-traveling ionospheric disturbances of ionograms observed during the 2011 Mw 9.0 Tohoku Earthquake. Earth Planets Space 63(7), 897-902. doi:10.5047/eps.2011.05.017.

Liu, J.Y., Tsai, Y.B., Chen, S.W., Lee, C.P., Chen, Y.C., Yen, H.Y., Chang, W.Y., Liu, C., 2006. Giant ionospheric disturbances excited by the M9.3 Sumatra earthquake of 26 December 2004. Geophys. Res. Lett. 33(2), L02103, doi:10.1029/2005GL023963.

Molchan, G.M., 1991. Structure of optimal strategies in earthquake prediction. Tectonophysics 193, 267276. doi:10.1016/0040-1951(91)90336-Q

Otsuka, Y., et al., 2006. GPS detection of total electron content variations over Indonesia and Thailand following the 26 December 2004 earthquake. Earth Planets Space 58(2), 159-165, doi:

10.1186/Bf03353373.

Rasouli, K., Hsieh, W., Cannon, A., 2012. Daily streamflow forecasting by machine learning methods with weather and climate inputs. J. Hydrol. 414, 284-293.

Stoica, P., Moses, R., 2005. Spectral Analysis of Signals. Upper Saddle River, Prentice Hall, New Jersey, USA.

Sun, Y.Y., Liu, J.Y., Lin, C.Y., Tsai, H.F., Chang, L.C., Chen, C.Y., et al., 2016. Ionospheric F-2 region perturbed by the 25 April 2015 Nepal earthquake. J. Geophys. Res. 121(6), 5778-5784. doi:10.1002/2015ja022280.

Sun, Y.Y., Oyama, K.I., Liu, J.Y., Jhuang, H.K., Cheng, C.Z., 2011. The neutral temperature in the ionospheric dynamo region and the ionospheric $\mathrm{F}$ region density during Wenchuan and Pingtung Doublet earthquakes. Nat. Hazards Earth Syst. Sci. 11, 1759-1768, doi:10.5194/nhess-11-1759-2011.

Szumilas, M., 2010. Explaining Odds Ratios. J. Can. Acad. Child Adolesc. Psychiatry 19, (3), 227-229.

Tan, A., Zhang, T.X., Wu, S.T., 2008. Pressure and density of air in mines. Indian j. radio space phys. 37, 64-67.

Tanimoto, T., Ishimaru, S., Alvizuri1, C., 2006. Seasonality in particle motion of microseisms. Geophys. J. Int. 166, 253-266, doi: 10.1111/j.1365-246X.2006.02931.x

Viera, A.J., 2008. Odds ratios and risk ratios: what's the difference and why does it matter?. South Med. J. $101,(7), 730-4$.

Wasilewski, S., 2014. Influence of barometric pressure changes on ventilation conditions in deep mines. Arch. Min. Sci., 59, 621-639. 


\section{Figures}
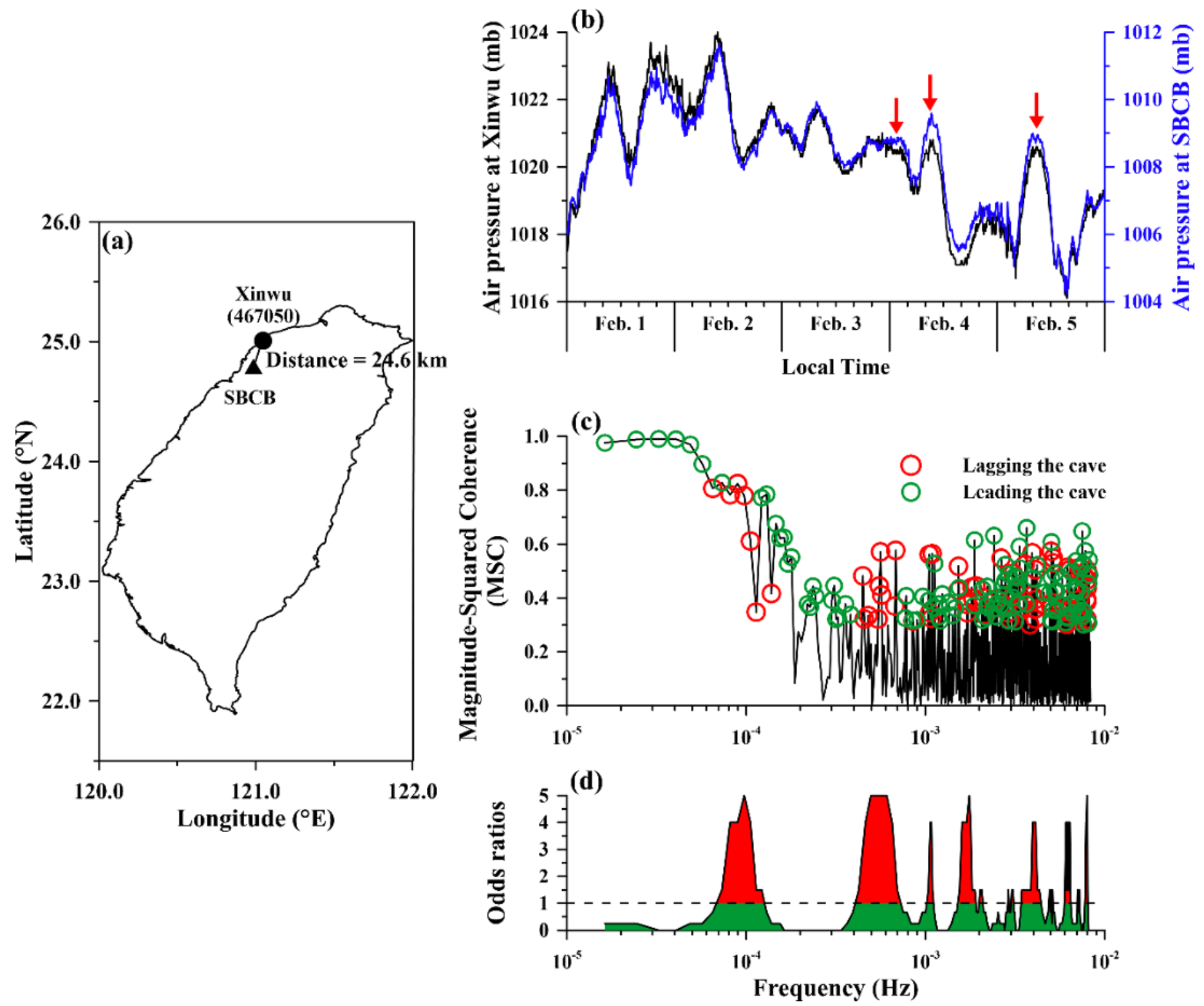

Figure 1

Locations and analytical results of air pressure data at Xinwu and SBCB stations. The locations of the two stations are shown in (a). The variations in air pressure during Feb. 1-5, 2016, are shown in (b). Black and blue lines denote the variations in air pressure at Xinwu and SBCB stations, respectively. Red arrows indicate the amplified variations in air pressure in the cave at SBCB station. The MagnitudeSquare Coherence (MSC) varied with frequency that is shown in (c). Green (red) open circles denote changes in air pressure at Xinwu station that lead (lag) to variations in the cave at SBCB station by 
utilizing the phase angle at each frequency. (d) The statistical results of the leading values with a coherence $>0.35$ determined by the Odds test with a moving window of 5 events.
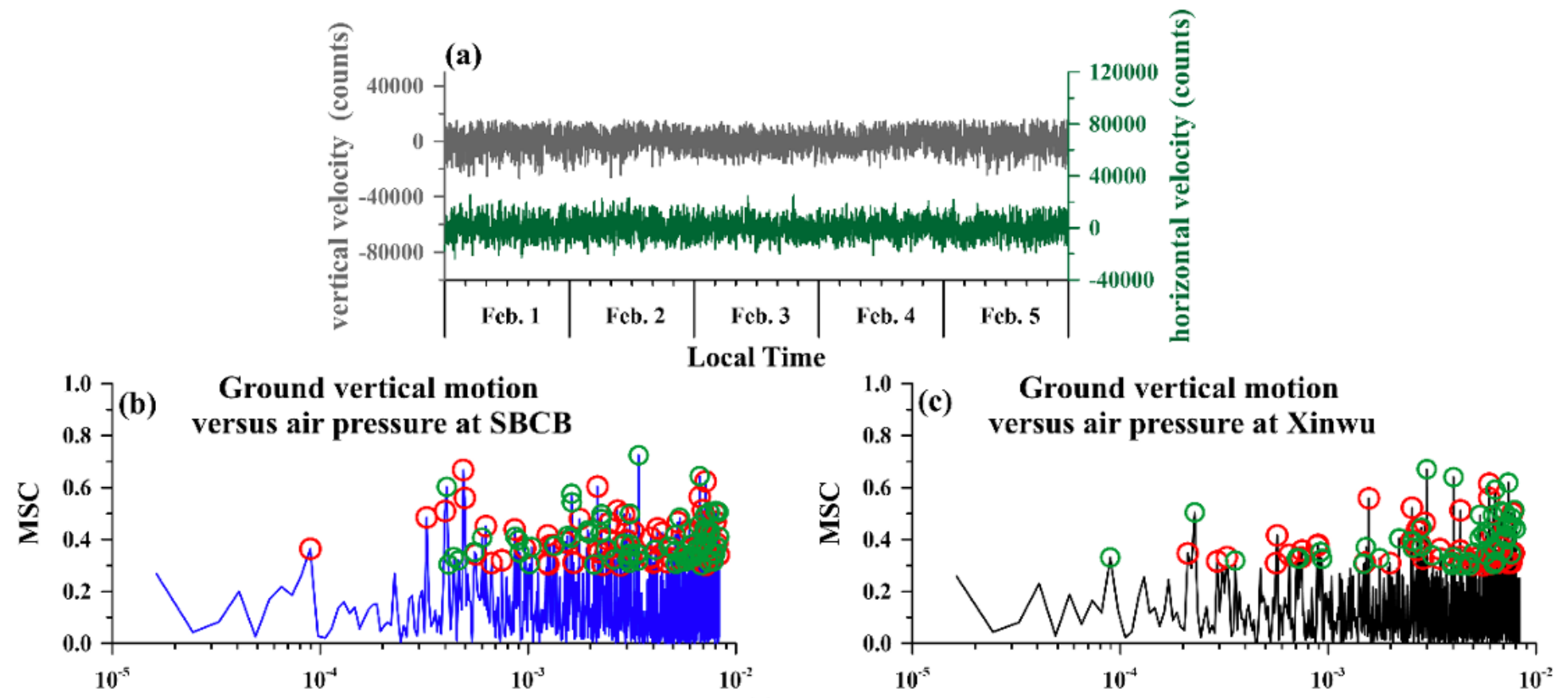

(c)
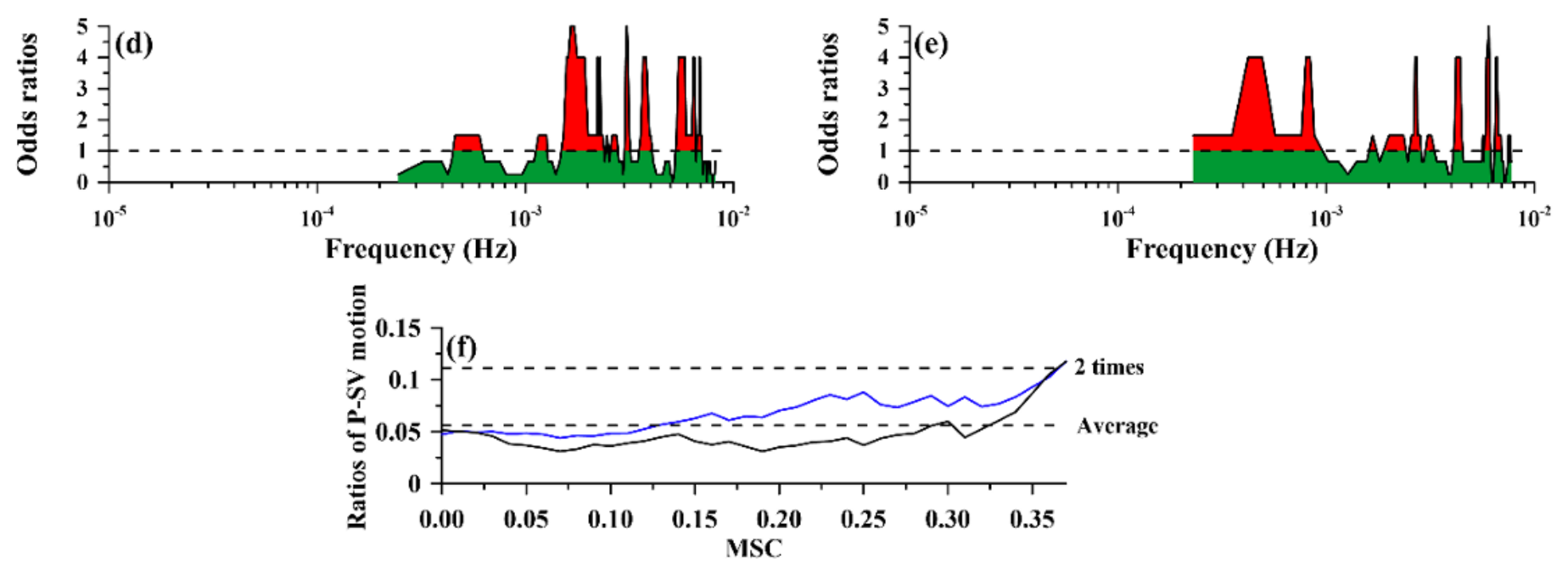

Figure 2

Variations in horizontal and vertical ground velocities and their comparison to air pressure data at Xinwu and SBCB stations. The variations in horizontal and vertical ground velocities at the SBCB station are shown in (a). The coherence between the vertical ground velocity and air pressure at the SBCB and Xinwu stations is shown in (b) and (c), respectively. Red (green) open circles denote that changes in ground velocity lead (lag) variations in air pressure. (d) and (e) The statistical results of the leading values for a coherence $>0.35$ in (b) and (c) determined by the Odds test using a moving window of 5 events. The Odds test reveals that the leading values are clustered at a few particular frequency bands. (f) The ratio of P-SV motion to the total coherence greater than the set criteria. The blue and black lines in (f) denote the ratio obtained using the air pressure data at the SBCB and Xinwu stations, respectively. 


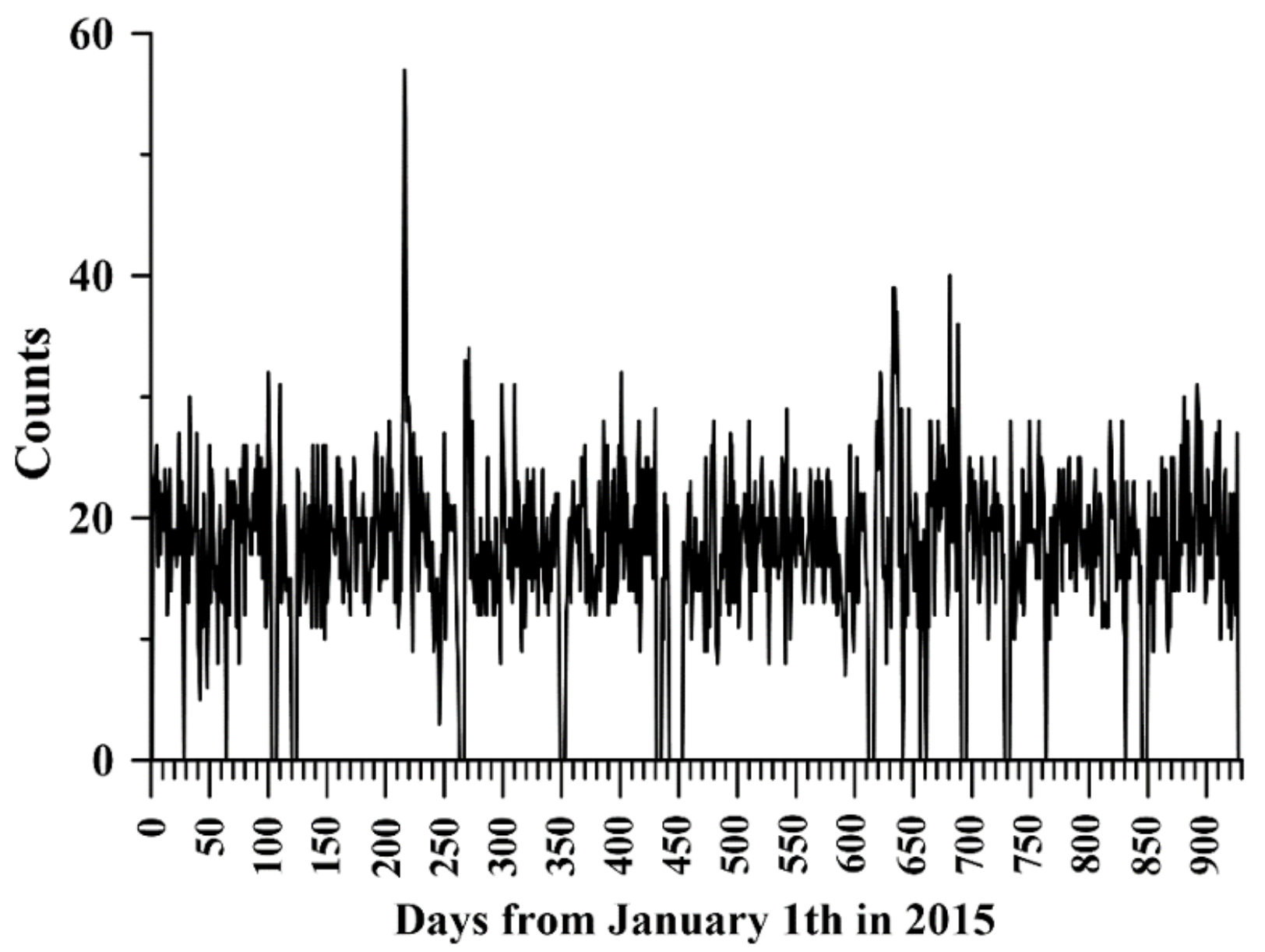

Figure 3

Daily counts of P-SV ground motion driving air pressure. The black line denotes the number of the P-SV waves at each frequency grid.

\section{Supplementary Files}

This is a list of supplementary files associated with this preprint. Click to download.

- Supplementary.docx 